Pré-Publicações do Departamento de Matemática

Universidade de Coimbra

Preprint Number 09-03

\title{
DESCENT FOR REGULAR EPIMORPHISMS IN BARR EXACT GOURSAT CATEGORIES
}

\author{
GEORGE JANELIDZE AND MANUELA SOBRAL \\ Dedicated to the memory of Gregory Maxwell Kelly
}

\begin{abstract}
We show that the category of regular epimorphisms in a Barr exact Goursat category is almost Barr exact in the sense that (it is a regular category and) every regular epimorphism in it is an effective descent morphism.
\end{abstract}

KEYWORDS: regular category, Barr exact category, Goursat category, equivalence relation, effective descent morphism.

AMS Subject Classification (2000): 18A20, 18A30, 18E10.

\section{Introduction}

Theory of Maltsev and Goursat categories is one of many small areas of category theory, where (in addition to several large areas!) Max Kelly made a significant contribution. In this short paper, dedicated to his memory, we add one more theorem to it, which is about the category $\operatorname{RE}(\mathcal{C})$ of regular epimorphisms in a Barr exact Goursat category $\mathcal{C}$. It says that every regular epimorphism in that category is an effective descent morphism. We then make a number of remarks explaining various motivations and connections of this result with known ones.

The standard reference for Goursat categories is the paper [1] of A. Carboni, G. M. Kelly, and M. C. Pedicchio. As explained there, Goursat categories are closely related to Maltsev categories in the sense of A. Carboni, J. Lambek, and M. C. Pedicchio [2]; see also much earlier work on "Maltsev conditions" of T. H. Fay in [3] and [4].

Received January 9, 2009.

Partially supported by South African NRF.

Partially supported by FCT/Centro de Matemática da Universidade de Coimbra. 
For a category $\mathcal{C}$ with finite limits, the category of equivalence relations in $\mathcal{C}$ will be denoted by $\operatorname{ER}(\mathcal{C})$. That is, an object $\mathrm{A}$ in $\operatorname{ER}(\mathcal{C})$ is a diagram

$$
A=\left(A_{1} \underset{a_{2}}{\stackrel{a_{1}}{\longrightarrow}} A_{0}\right)
$$

in $\mathcal{C}$, which is the underlying graph of an internal groupoid with $a_{1}$ and $a_{2}$ jointly monic. A morphism $f: A \rightarrow B$ is a pair $\left(f_{0}: A_{0} \rightarrow B_{0}, f_{1}: A_{1} \rightarrow B_{1}\right)$ of morphisms in $\mathcal{C}$ with $f_{0} a i=b_{i} f_{1}(i=1,2)$. When $\mathcal{C}$ is Barr exact, the category $\operatorname{ER}(\mathcal{C})$ is of course equivalent to the category $\operatorname{RE}(\mathcal{C})$ of regular epimorphisms in $\mathcal{C}$.

Proposition 6.5 of [1] implies the following:

Theorem 1.1. The following conditions on a regular category $\mathcal{C}$ are equivalent:

(a) $\mathcal{C}$ is a Goursat category;

(b) for every diagram

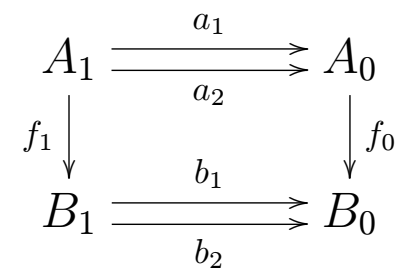

in $\mathcal{C}$, in which $f_{0}$ and $f_{1}$ are regular epimorphisms, $f_{0} a_{i}=b_{i} f_{1}(i=1,2)$, $\left(a_{1}, a_{2}\right)$ determines an equivalence relation, and $b_{1}$ and $b_{2}$ are jointly monic, $\left(b_{1}, b_{2}\right)$ also determines an equivalence relation.

This theorem is in fact all we need to know about Goursat categories for the purposes of the present paper.

Let us also recall (see e.g. [9] or [8], for alternative definitions, various explanations and proofs, although most of them were known long before; see A. H. Roques $\mathrm{PhD}$ Thesis [11] for the last part of 1.3(b) and much more general results):

Definition 1.2. A morphism $p: E \rightarrow B$ in a category $\mathcal{C}$ with finite limits is said to be an effective (global) descent morphism if the pullback functor $p^{*}: \mathcal{C} \downarrow B \rightarrow \mathcal{C} \downarrow E$ is monadic.

Theorem 1.3. (a) Every effective descent morphism in a category with finite limits and coequalizers of equivalence relations is a regular epimorphism. 
(b) Every regular epimorphism in a Barr exact category is an effective descent morphism, and the same is true for the category of reflexive relations in a Barr exact category.

(c) Let $p: E \rightarrow B$ be a morphism in a category $\mathcal{C}$ with finite limits, which is a full subcategory closed under finite limits in another such category $\mathcal{D}$. If $p$ is an effective descent morphism in $\mathcal{D}$, then it is an effective descent morphism in $\mathcal{C}$ if and only if for every pullback diagram in $\mathcal{D}$ of the form

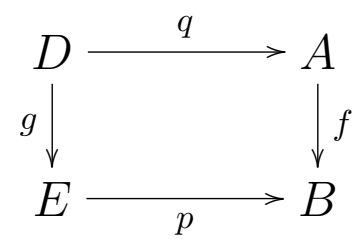

we have $D \in \mathcal{C} \Rightarrow A \in \mathcal{C}$.

\section{The descent theorem}

Throughout this section $\mathcal{C}$ denotes a fixed Barr exact Goursat category. The category $\operatorname{ER}(\mathcal{C})$ of equivalence relations in $\mathcal{C}$ is a full subcategory in the category $\operatorname{RR}(\mathcal{C})$ of reflexive relations in $\mathcal{C}$, which itself is a full subcategory in the Barr exact category $\operatorname{RG}(\mathcal{C})$ of reflexive graphs in $\mathcal{C}$. We also know that a morphism $f: A \rightarrow B$ in $\operatorname{RR}(\mathcal{C})$ is a regular epimorphism if and only if its components $f_{0}: A_{0} \rightarrow B_{0}$ and $f_{1}: A_{1} \rightarrow B_{1}$ are regular epimorphisms in $\mathcal{C}$. Together with Theorem 1.1 this gives:

Lemma 2.1. (a) If $f: A \rightarrow B$ is a morphism in $E R(\mathcal{C})$, and

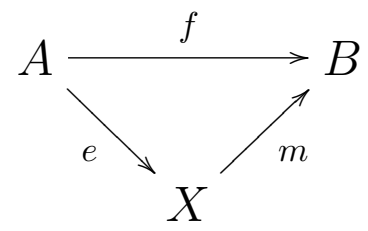

is its (regular epi, mono)-factorization in $R R(\mathcal{C})$, or equivalently in $R G(\mathcal{C})$, then $X$ is in $E R(\mathcal{C})$

(b) A morphism $f: A \rightarrow B$ in $E R(\mathcal{C})$ is a regular epimorphism if and only if it is a regular epimorphism in $R R(\mathcal{C})$, or equivalently in $R G(\mathcal{C})$.

(c) In particular $E R(\mathcal{C})$ is a regular category, and the inclusion functors $E R(\mathcal{C}) \rightarrow R R(\mathcal{C}) \rightarrow R G(\mathcal{C})$ preserve finite limits, regular epimorphisms, and (regular epi, mono)-factorizations.

Lemma 2.2. Every regular epimorphism in $E R(\mathcal{C})$ is an effective descent morphism. 
Proof: For a regular epimorphism $p: E \rightarrow B$ in $\operatorname{ER}(\mathcal{C})$, consider the diagram

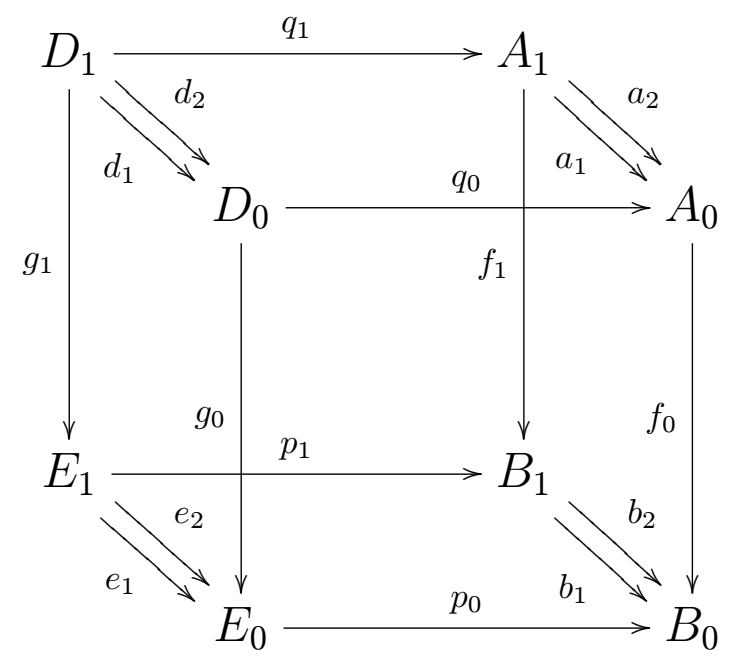

which displays a pullback of the form $(2)$ in $R R(\mathcal{C})$. Since $p$ is a regular epimorphism in $\operatorname{ER}(\mathcal{C})$, it is a regular epimorphism in $R R(\mathcal{C})$ by Lemma $2.1(\mathrm{~b})$, and therefore it is an effective descent morphism in $\mathrm{RR}(\mathcal{C})$ by Theorem 1.3(b). After that, according to Theorem 1.3(c), all we need to prove is that if $\left(d_{1}, d_{2}\right)$ determines an equivalence relation, then the same is true for $\left(a_{1}, a_{2}\right)$. However, this follows from Theorem 1.1(b) since $q_{0}$ and $q_{1}$ being pullbacks of $p_{0}$ and $p_{1}$ respectively are regular epimorphisms.

Let us translate this result into the language of regular epimorphisms using the category equivalence $\operatorname{ER}(\mathcal{C}) \sim \operatorname{RE}(\mathcal{C})$. Since we used (1) do display equivalence relations, we should now display objects in $\operatorname{RE}(\mathcal{C})$ as

$$
A=\left(A_{0} \stackrel{a}{\longrightarrow} A_{-1}\right) .
$$

Theorem 2.3. The following conditions on a morphism $p: E \rightarrow B$ in $R E(\mathcal{C})$ are equivalent:

(a) $p$ is an effective descent morphism;

(b) $p$ is a regular epimorphism;

(c) $p_{0}: E_{0} \rightarrow B_{0}$ and the induced morphism $p_{0} \times p_{0}: E_{0} \times_{E_{-1}} E_{0} \rightarrow$ $B_{0} \times_{B_{-1}} B_{0}$ are regular epimorphisms in $\mathcal{C}$;

(d) the morphism $p_{0} \times p_{0}: E_{0} \times_{E_{-1}} E_{0} \rightarrow B_{0} \times_{B_{-1}} B_{0}$ is a regular epimorphism in $\mathcal{C}$.

Proof: Since the categories $\operatorname{ER}(\mathcal{C})$ and $\operatorname{RE}(\mathcal{C})$ are equivalent, $(\mathrm{a}) \Leftrightarrow(\mathrm{b})$ follows from Lemma 2.2 and Theorem 1.3(a). (b) $\Leftrightarrow(\mathrm{c})$ follows from Lemma 2.1(b) 
since the morphism in $\operatorname{ER}(\mathcal{C})$ corresponding to $p$ displays as

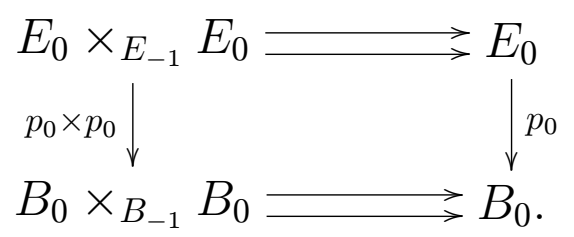

$(\mathrm{c}) \Leftrightarrow(\mathrm{d})$ follows from the fact that $\mathcal{C}$ is a regular category.

\section{Remarks}

3.1. Theorem 2.3 should first of all be compared of course with the description of effective descent morphisms in $\operatorname{RE}(\mathcal{S}$ ets $) \sim \mathrm{ER}(\mathcal{S}$ ets $)$ : just as for (finite) preorders in [7], the following conditions on a morphism $p: E \rightarrow B$ in $\operatorname{RE}(\mathcal{S}$ ets $)$ are equivalent:

(a) $p$ is an effective descent morphism;

(b) $p_{0}: E_{0} \rightarrow B_{0}$ and the induced maps $p_{0} \times p_{0}: E_{0} \times_{E_{-1}} E_{0} \rightarrow B_{0} \times_{B_{-1}} B_{0}$ and $p_{0} \times p_{0} \times p_{0}: E_{0} \times_{E_{-1}} E_{0} \times_{E_{-1}} E_{0} \rightarrow B_{0} \times_{B_{-1}} B_{0} \times_{B_{-1}} B_{0}$ are surjective;

(c) the map $p_{0} \times p_{0} \times p_{0}: E_{0} \times_{E_{-1}} E_{0} \times_{E_{-1}} E_{0} \rightarrow B_{0} \times_{B_{-1}} B_{0} \times_{B_{-1}} B_{0}$ is surjective.

Note also that (from [7] or directly), in $\operatorname{ER}(\mathcal{S}$ ets) we have:

\{reg. epimorphisms $\} \subset\{$ pullback stable reg. epimorphisms $\}$

$\subset$ effective descent morphisms

with all the inclusions strict, while in the Goursat case these three classes of morphisms coincide with each other.

3.2. We do not know how to describe effective descent morphisms in $\operatorname{ER}(\mathcal{C})$ $\sim \operatorname{RE}(\mathcal{C})$ for an arbitrary Barr exact category $\mathcal{C}$. However, condition 3.1(b) can still be used (with regular epimorphisms instead of surjections) as a sufficient condition, which follows from much more general results of I. Le Creurer [10]. As also follows from results of [10], it becomes necessary (and sufficient) when $\mathcal{C}$ is a pretopos. Moreover, Le Creurer has fully described effective descent morphisms of internal categories in a lextensive category in [10]. This question is still to be investigated in the Goursat (instead of lextensive) case; in the Barr exact Maltsev case it becomes trivial since in that case the category of internal categories becomes Barr exact, as shown by M. Gran [5]. 
3.3. When $\mathcal{C}$ is semi-abelian in the sense of [6], the equivalent conditions of Theorem 2.3 are also equivalent to any of the following two conditions:

(a) the morphisms $p_{0}$ and $<p r_{1}, p_{0} \times p_{0}>: E_{0} \times_{E_{-1}} E_{0} \rightarrow E_{0} \times_{B_{0}}\left(B_{0} \times_{B_{-1}} B_{0}\right)$ are regular epimorphisms;

(b) the morphism $p_{0}$ and the morphism $\operatorname{Ker}(e) \rightarrow \operatorname{Ker}(b)$ induced by $p_{0}$ are regular epimorphisms (using the notation (3)).

The finite preorder/topological version of the implication $2.3(\mathrm{a}) \Rightarrow 3.3(\mathrm{a})$ would be "every quotient map is open", which again shows the difference with the case of $\operatorname{RE}(\mathcal{S} e t s)$.

3.4. When $\mathcal{C}$ is semi-abelian the categories $\operatorname{ER}(\mathcal{C}) \sim \operatorname{RE}(\mathcal{C})(=\operatorname{NE}(\mathcal{C})$, the category of normal epimorphisms in $\mathcal{C}$ ) are equivalent to the category $\operatorname{NM}(\mathcal{C})$ of normal monomorphisms in $\mathcal{C}$. Remark 3.3(b) then tells us that the inclusion of $\operatorname{NM}(\mathcal{C})$ into the category $\mathrm{M}(\mathcal{C})$ of all monomorphisms in $\mathcal{C}$ has the same properties as the inclusion $\operatorname{ER}(\mathcal{C}) \rightarrow \operatorname{RR}(\mathcal{C})$. In particular a morphism in $\operatorname{NM}(\mathcal{C})$ is an effective descent morphism if and only if it is an effective descent morphism in $\mathrm{M}(\mathcal{C})$. When $\mathcal{C}$ abelian, and so all monomorphisms and epimorphisms in $\mathcal{C}$ are normal, we have category equivalences

$\{$ epimorphisms $\} \sim\{$ short exact sequences $\} \sim\{$ monomorphisms $\}$

and the normal $=$ regular epimorphisms in these categories form the main example of a structure called quasi-abelian category by N. Yoneda in his classical work [12].

3.5. The way Theorem 1.1, i.e. in fact Proposition 6.5 of [1], is used in the proof of Lemma 2.2 suggests to ask if Theorem 2.3 actually characterizes Barr exact Goursat categories. It seems, however, that this is not the case as e.g. not every quasivariety of universal algebras in which every regular epimorphism is an effective descent morphism is a variety. The most interesting "concrete" problem here seems to be to characterize varieties of universal algebras satisfying Theorem 2.3, and give an example of a non-Goursat variety with this property.

\section{References}

[1] A. Carboni, G. M. Kelly, and M. C. Pedicchio, Some remarks on Maltsev and Goursat categories, Appl. Categ. Structures1, 1993, 385-421.

[2] A. Carboni, J. Lambek, and M.C. Pedicchio, Diagram chasing in Malcev categories, J. Pure Appl. Algebra 69, 1990, 271-284. 
[3] T. H. Fay, On commuting congruences in regular categories, Math. Colloq. Univ. Cape Town11, 1977, 13-31.

[4] T. H. Fay, On categorical conditions for congruences to commute, Algebra Universalis 8, 1978, 173-179.

[5] M. Gran, Internal categories in Malcev categories, J. Pure Appl. Algebra, 143, 1999, 221-229.

[6] G. Janelidze, L. Márki, and W. Tholen, Semi-abelian categories, J. of Pure Appl. Algebra 168, $2002,367-386$.

[7] G. Janelidze and Sobral, Finite preorders and topological descent I, J. Pure Appl. Algebra 175(1-3), 2002, 187-205.

[8] G. Janelidze, M. Sobral and W. Tholen, Beyond Barr exactness: effective descent morphisms, Categorical Foundations; Special Topics in Order, Topology, Algebra, and Sheaf Theory, Cambridge University Press, 2004, 359-405.

[9] G. Janelidze and W. Tholen, Facets of Descent I, Appl. Categ. Structures 2, 1994, 245-281.

[10] I. Le Creurer, Descent of internal categories, PhD Thesis, Louvain-la-Neuve, 1999.

[11] A. H. Roque, Grothendieck descent in quasi-varieties of algebraic and relational structures, PhD Thesis, Aveiro University (Portugal), 2004.

[12] N. Yoneda, On Ext and exact sequences, J. Fac. Sci. Tokyo 18, 1960, 507-576.

George Janelidze

Dept. of Math. and Appl. Math., University of Cape Town, Rondebosh 7701, Cape Town, South AFrica

E-mail address: janelidg@maths.uct.ac.za

Manuela Sobral

CMUC, Department of Mathematics, University of Coimbra, 3001-454 Coimbra, Portugal

E-mail address: sobral@mat.uc.pt 